\title{
MeCP2-Mediated Transcription Repression in the Basolateral Amygdala May Underlie Heightened Anxiety in a Mouse Model of Rett Syndrome
}

\author{
Megumi Adachi, Anita E. Autry, Herb E. Covington III, and Lisa M. Monteggia \\ Department of Psychiatry, The University of Texas Southwestern Medical Center, Dallas, Texas 75390-9070
}

\begin{abstract}
Rett syndrome (RTT) is an X-linked neurodevelopmental disorder that results from loss of function mutations in the methyl-CpG binding protein 2 (MECP2) gene. Using viral-mediated basolateral amygdala (BLA)-specific deletion of Mecp2 in mice, we show that intact Mecp2 function is required for normal anxiety behavior as well as some types of learning and memory. To examine whether these behavioral deficits are the result of impaired transcriptional repression, because $M e c p 2$ is believed to act as a transcriptional repressor in complex with histone deacetylases (HDACs), we infused a HDAC inhibitor chronically into the BLA of wild-type mice. We found that HDAC inhibition produces behavioral deficits similar to those observed after the deletion of Mecp2 in the BLA. These results suggest a key role for Mecp2 as a transcriptional repressor in the BLA in mediating behavioral features of RTT.
\end{abstract}

\section{Introduction}

Rett syndrome (RTT) is a neurodevelopmental disorder caused by mutations in the gene encoding methyl-CpG binding protein 2 (MECP2) that are predicted to result in loss of function of the protein (MeCP2) (Amir et al., 1999). Individuals affected with RTT are born healthy and experience normal development until 6-18 months of age, after which they enter a period characterized by regression of motor and language skills (Hagberg et al., 1983). RTT patients display a range of phenotypes that are predominantly expressed in the CNS, including stereotypical hand movements, problems with gait, social behavioral deficits associated with autistic-like features, heightened anxiety, and loss of cognitive abilities including some learning disabilities (Hagberg et al., 1983; Sansom et al., 1993; Mount et al., 2002; Weaving et al., 2005).

$\mathrm{MeCP} 2$ is expressed at high levels in postmitotic neurons and only low levels in immature neurons, although RTT is a neurodevelopmental disorder (Shahbazian et al., 2002b; Kishi and Macklis, 2004; Mullaney et al., 2004). So why this apparent paradox? Recent work has demonstrated that loss of Mecp2 function in postmitotic cortical and hippocampal neurons produces deficits in synaptic function and plasticity (Dani et al., 2005; Asaka et al., 2006; Moretti et al., 2006; Nelson et al., 2006, 2008); however, it is currently unknown whether these deficits underlie the behavioral features of RTT.

Received Sept. 2, 2008; revised Jan. 29, 2009; accepted Feb. 24, 2009

This work was funded by National Institute of Mental Health Grant MH077944 (L.M.M.) and a postdoctoral fellowship from the International Rett Syndrome Foundation (M.A.). We thank Dr. S. Chakravarty for the AAV preparations. We acknowledge Dr. S. Birnbaum and A. Graham for assistance with the behavioral testing. We thank Drs. M. Colledge and E. Kavalali for critically reading this manuscript and for helpful discussions.

Correspondence should be addressed to Lisa M. Monteggia, Department of Psychiatry, The University of Texas Southwestern Medical Center, 5323 Harry Hines Boulevard, Dallas, TX75390-9070.E-mail: lisa.monteggia@utsouthwestern.edu. DOI:10.1523/JNEUROSCI.4225-08.2009

Copyright $\odot 2009$ Society for Neuroscience $\quad 0270-6474 / 09 / 294218-10 \$ 15.00 / 0$
Attempts to model the disease by generating constitutive Mecp 2 knock-out (KO) mice result in the recapitulation of many of the neurological symptoms of RTT. However, these mice die early in postnatal development (Chen et al., 2001; Guy et al., 2001), which prevents their use in behavioral characterization studies. To circumvent these potential problems, conditional $\mathrm{KO}$ mice, in which floxed Mecp2 mice were crossed with calcium/ calmodulin-dependent protein kinase II-Cre recombinase transgenic mice to selectively delete Mecp2 in forebrain regions (cortex, hippocampus, striatum, and amygdala), were generated (Chen et al., 2001). We have recently characterized these conditional Mecp2 $\mathrm{KO}$ mice in a wide array of behavioral tests and found that these mice have many of the behavioral abnormalities that are reminiscent of the symptoms seen in RTT patients, including impaired motor coordination, increased anxiety, abnormal social interaction with other mice, and deficits in cuedependent fear conditioning (Gemelli et al., 2006). Although these data suggest that the general loss of Mecp2 in the forebrain may underlie many of the behavioral symptoms exhibited by RTT patients, it remains unclear which specific brain regions or neural pathways mediate these abnormalities. Given the importance of the basolateral amygdala (BLA) in the perception and modulation of emotion, including fear, stress, and anxiety (Campeau and Davis, 1995; Maren and Fanselow, 1995; Wilensky et al., 1999; Davis and Shi, 2000; LeDoux, 2000; Roozendaal et al., 2002; Rainnie et al., 2004; LeDoux, 2007), we examined whether the loss of Mecp2 in the BLA was sufficient for the development of many of the behavioral phenotypes exhibited by RTT patients. To this end, we used a viral-mediated approach to specifically delete Mecp2 in the BLA and show that Mecp2 is required for normal anxiety behavior as well as some types of learning and memory. Our data further suggest that Mecp2 may be mediating these behavioral effects via its role as a transcriptional repressor. 


\section{Materials and Methods}

Adeno-associated virus injection. To inject the addeno-associated virusgreen fluorescent protein (AAV-GFP) or AAV-CreGFP, floxed Mecp2 mice (3- to 5-month-old males) were subjected to stereotaxic surgery as described previously (Adachi et al., 2008). The floxed Mecp2 mouse line (Chen et al., 2001) was on a mixed 129/BALB/c background that was backcrossed to C57BL/6 mice for nine generations. The coordinates relative to bregma were anteroposterior, $-0.6 \mathrm{~mm}$; lateral, $+3.3 \mathrm{~mm}$; dorsoventral, $-4.5 \mathrm{~mm}$. All mice were injected bilaterally into the BLA with either AAV-GFP or AAV-CreGFP. The use of GFP allowed for the infected neurons to be visualized. Previous work had demonstrated that the GFP did not interfere with Cre recombinase activity (Berton et al., 2006; Adachi et al., 2008). To achieve maximal expression of Cre recombinase and excision of Mecp2 gene, the mice were group-housed (3-4 per cage) for $16 \mathrm{~d}$ after the surgery until the commencement of the behavioral experiments. All experiments were approved by the University of Texas Southwestern Medical Center Animal Care and Use Committee.

Fluorescent in situ hybridization. After the completion of the behavioral tasks, the mice were killed by rapid decapitation, and the brains were dissected out, rapidly frozen on dry ice, and stored at $-80^{\circ} \mathrm{C}$. The brains were sectioned at $14 \mu \mathrm{m}$ thickness on a cryostat, collected on coated glass slides, and subjected to fluorescent in situ hybridization (FISH). Mecp2 and Cre recombinase probes were prepared by in vitro transcription and labeled with digoxigenin and fluorescein, respectively. The sequences for the probes were listed by Adachi et al. (2005). The detailed methods were described previously (Adachi et al., 2008). The FISH analyses allowed us to examine the bilateral injection sites and confirm whether the placement was in the correct location. If a placement was not correct bilaterally in the BLA for an injection, the behavioral data of the animal were disregarded.

Quantitative reverse transcription PCR. To determine the relative amount of Mecp2 expression in BLA after stereotaxic injections, we used a quantitative reverse transcription (QRT)-PCR approach. The mice were killed by rapid decapitation, and the brains were sectioned at $10 \mu \mathrm{m}$ thickness and collected on polyethylene naphthalate (PEN) membranecoated glass slides (Leica). Sections were briefly dehydrated in 70, 90, and $100 \%$ ethanol and then subjected to laser microdissection using an AS LMD system (Leica). The entire BLA containing GFP-positive cells was dissected from each section. Seven to nine sections were pooled to extract total RNA using a PicoPure RNA isolation kit (Arcturus). Extracted RNA was treated with DNase I for $30 \mathrm{~min}$ at $37^{\circ} \mathrm{C}$ and reverse transcribed by using random hexamers and Superscript III (Invitrogen) to synthesize single-stranded cDNA. Using cDNA as a template, transcripts for Mecp2, Cre recombinase, and $18 \mathrm{~S}$ ribosomal RNA (rRNA) were amplified using Power SYBR Green PCR master mix (Applied Biosystems) in a Mx3000P QPCR system (Stratagene). The thermal cycling conditions for PCR amplification consisted of 1 cycle of $95^{\circ} \mathrm{C}$ for $10 \mathrm{~min}, 40$ cycles of $95^{\circ} \mathrm{C}$ for $30 \mathrm{~s}, 60^{\circ} \mathrm{C}$ for $20 \mathrm{~s}$, and $72^{\circ} \mathrm{C}$ for $30 \mathrm{~s}$, and $1 \mathrm{cycle}$ of $95^{\circ} \mathrm{C}$ for $1 \mathrm{~min}$ and $55^{\circ} \mathrm{C}$ for $30 \mathrm{~s}$. Primers used for Cre and Mecp 2 were listed by Adachi et al. $(2005,2008)$. The primers for $18 \mathrm{~S}$ rRNA are 5'-GCC GCT AGA GGT GAA ATT CTT G- $3^{\prime}$ and $5^{\prime}$-CAT TCT TGG CAA ATG CTT TCG- ${ }^{\prime}$. The fold change in Cre and Mecp2expression relative to $18 \mathrm{~S}$ rRNA was calculated as mean \pm SEM.

Infusion of the histone deacetylase inhibitor. Male C57BL/6 mice that were $\sim 8$ weeks of age were used. Osmotic pumps (Alzet) were filled with either vehicle (5\% cyclodextrin in PBS) or suberoylanilide hydroxamic acid (SAHA) (100 $\mu \mathrm{M}$ in vehicle solution). The pumps were connected to bilateral cannulae via vinyl tubes and placed under the back of the animals. The cannulae (Plastics One) were then positioned at $-1.5 \mathrm{~mm}$ anteroposterior from bregma to provide bilateral infusion of drug into the BLA. After the surgery, the animals were singly housed, handled daily for acclimation, and allowed to recover until commencement of the behavioral tests. At the completion of the experiment, all animals were killed and the brains were dissected out and cut in half, laterally. From one half of the brain, BLA was dissected out to prepare protein extracts for Western blot analyses. The other half of the brain was placed in $4 \%$ paraformaldehyde for $72 \mathrm{~h}$, processed in $20 \%$ glycerol overnight, sectioned at $30 \mu \mathrm{m}$ thickness, and stained with Nissl to verify the placement of the cannulae. If the placement was incorrect, the data points from that animal were excluded from analyses.

Western blot analysis. Proteins were extracted in lysis buffer composed of (in mM): 25 HEPES, pH 7.9, $150 \mathrm{NaCl}, 1$ PMSF, $20 \mathrm{NaF}, 1$ DTT, $0.1 \%$ NP40, and proteinase inhibitor cocktails (Sigma). Twenty micrograms of proteins were resolved on a $12.5 \%$ SDS-PAGE gel and transferred to a nitrocellulose membrane. Dilutions of primary antibodies were 1:1000 for both AcH3 and AcH4 antibodies (Millipore), 1:2000 for MeCP2 (Affinity Bioreagents), and 1:10,000 for glyceraldehyde-3-phosphate dehydrogenase (GAPDH) (Cell Signaling Technology) and actin (MP Biomedicals). Signals were detected using an enhanced chemiluminescence system (GE Healthcare Bio-Sciences).

Behavioral overview. Mice were on a $12 \mathrm{~h}$ light/dark cycle with ad libitum food and water. Behavioral testing was done on male floxed Mecp2 mice starting 2 weeks after AAV-GFP or AAV-CreGFP injections or male C57BL/ 6 mice $8 \mathrm{~d}$ after SAHA infusions. At the completion of the behavioral experiments, the regional specificity of the injection into the BLA region for the viral-injected animals and the histone deacetylase (HDAC)-infused animals were verified. If the placements were outside the BLA, the behavioral data from that animal were removed from analysis.

Before all testing, mice were allowed to habituate in the behavioral room for $1 \mathrm{~h}$. For all experiments, data were presented as mean \pm SEM, and Student's $t$ test was used to analyzed data with significance set as $p<$ 0.05 unless otherwise noted.

Rotarod and locomotor activity. The rotarod test was performed as described previously (Gemelli et al., 2006). For locomotor activity, animals were placed in a fresh home cage and locomotor activity was measured for $1 \mathrm{~h}$ by photocell beams linked to computer data acquisition software (San Diego Instruments). Data were analyzed with repeated ANOVA. Data are presented as mean \pm SEM.

Open-field test. Mice were placed in a $72 \mathrm{~cm}^{2}$ open field under dim lighting, and their activity was monitored for $5 \mathrm{~min}$ with a video tracking system using EthoVision software (Noldus Information Technology). The time spent in the center of the open field $(14 \times 14 \mathrm{~cm})$, latency to enter the center, and frequency to enter the center were analyzed. Peripheral zones are $5 \mathrm{~cm}$ around perimeter of the arena and complete center is $62 \times 62 \mathrm{~cm}$.

Elevated-plus maze. Mice were placed in the center of a maze shaped in a cross (each arm $33 \mathrm{~cm} \times 5 \mathrm{~cm}$ ) that was elevated 2 feet above the floor with two open arms and two closed arms (25-cm-tall walls on the closed arms). The behavior of the mice was monitored for $5 \mathrm{~min}$ with a video tracking system, and the time spent in the closed and open arms, as well as the latency to enter open-arms, was determined by using EthoVision software.

Social interaction test. In this paradigm, we measured social interest, which was defined as the approach that an experimental mouse made toward a novel male C57BL/6 mouse (target). An experimental mouse was placed in an open-field arena $(42 \mathrm{~cm} \times 42 \mathrm{~cm})$ containing a wire mesh cage located against one of the walls, and its movements were recorded with a video tracking system using EthoVision software for 5 min. Immediately thereafter, the experimental mouse was removed from the arena and the target mouse was placed in the wire mesh cage. The experimental mouse was then reintroduced in the arena. The movement of the experimental mouse was recorded for another $5 \mathrm{~min}$. The wire mesh cage allowed visual and olfactory interactions between the mice, but prevented direct physical contact. Using the video tracking system, the duration of time spent in the interaction zone in the absence and presence of the target was measured.

Fear conditioning. The fear-conditioning paradigm was assessed as previously described (Monteggia et al., 2004). Briefly, mice were placed in individual chambers for $2 \mathrm{~min}$ followed by a loud tone ( $90 \mathrm{~dB}$ ) for $30 \mathrm{~s}$, immediately followed by a $0.5 \mathrm{~mA}$ footshock for $2 \mathrm{~s}$. Mice remained in the box for $1 \mathrm{~min}$, at which time they again received a loud tone $(90 \mathrm{~dB})$ for $30 \mathrm{~s}$ and then an immediate $0.5 \mathrm{~mA}$ footshock for $2 \mathrm{~s}$. The mice were immediately removed and placed back into their home cages. Each chamber was cleaned with $70 \%$ ethanol between animals. To test for context-dependent fear conditioning, $24 \mathrm{~h}$ later the mice were placed back in the same boxes without a tone or shock. The amount of time the animal spent freezing was scored by an observer blind to the genotype. Freezing behavior was defined as no movement except for respiration. 
Four hours later, the cue test was performed. To test for cue-dependent fear conditioning, mice were placed in a novel environment with no tone or shock for $3 \mathrm{~min}$ followed by $3 \mathrm{~min}$ of the tone. The amount of time the mice spent freezing was assessed as described above.

Pain sensitivity test. Pain sensitivity was assessed as previously described (Gemelli et al., 2006). Responses to foot shock were measured 1 week after the fear-conditioning test to allow the mice to extinguish from the context. Footshock started at $0.0 \mathrm{~mA}$ and increased by 0.05 $\mathrm{mA}$ every $20 \mathrm{~s}$ to $0.25 \mathrm{~mA}$.

\section{Results}

Characterization of the localized Mecp2 deletion in BLA

We used a viral-mediated approach to delete Mecp 2 selectively in the BLA and then examined the behavioral profile of the mice (Fig. $1 A)$. We combined the AAV expression system with the Cre/loxP site-specific recombination system as previously described (Berton et al., 2006; Adachi et al., 2008). Floxed Mecp2 mice were bilaterally injected with AAV expressing a fusion construct of Cre recombinase and GFP (AAV-CreGFP) to produce the regionally specific deletion, or as a control AAV expressing GFP (AAV-GFP) (Fig. 1B). Floxed Mecp2 mice used in this study carry loxP sites flanking exon 3 of the Mecp2 gene, which encodes a majority of the methyl-DNA binding domain of $\mathrm{MeCP} 2$. Previous studies using this floxed Mecp2 mouse line have demonstrated the efficient deletion of Mecp2 on expression of Cre recombinase (Chen et al., 2001; Gemelli et al., 2006).

To determine the regional specificity of our placements after viral injections, we used epifluorescence microscopy to confirm GFP expression in the BLA (data not shown), followed by double FISH to evaluate Cre recombinase and Mecp2 expression. Floxed Mecp2 mice receiving AAVGFP with confirmed viral placement bilaterally into BLA had no detectable expression of Cre recombinase (Fig. 1C). In contrast, floxed Mecp2 mice receiving bilateral injections of AAV-CreGFP into the BLA had strong Cre mRNA expression

(Fig. 1D). We found robust expression of Mecp2 mRNA in the BLA of control AAV-GFP-injected animals, whereas we found a dramatic reduction of Mecp2 mRNA expression in the BLA of AAV-CreGFP-injected mice, demonstrating our ability to selectively delete the Mecp2 gene in a regionally specific manner (Fig. $1 C, D)$. To confirm that the reduction in Mecp2 expression in the AAV-CreGFP-injected mice was not caused by cell loss, we used $4^{\prime}, 6^{\prime}$-diamidino-2-phenylindole dihydrochloride (DAPI) staining to label cell nuclei and did not detect a difference in cell density between AAV-CreGFP- and AAV-GFP-injected mice (Fig. 1C,D).

To quantify the reduction in Mecp2 expression after AAVCreGFP, the BLA was laser-microdissected from several brain sections encompassing the BLA, and QRT-PCR was performed (Fig.

A

cox

E
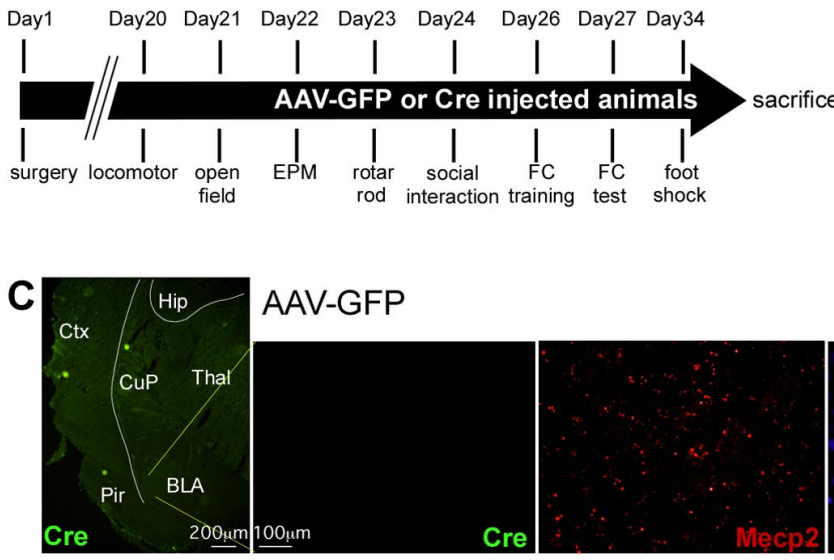

B
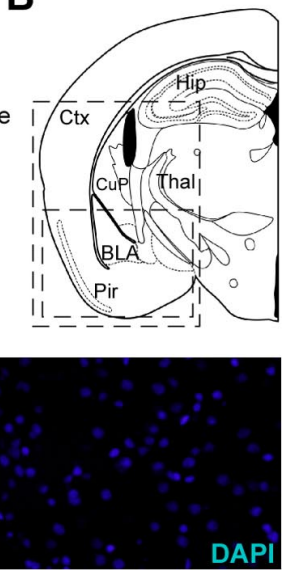

Figure 1. AAV-CreGFP-mediated deletion of Mecp2 from BLA neurons. $A$, Experimental timeline for the behavioral experiments. $\boldsymbol{B}$, A schematic of a mouse brain coronal section at $-1.94 \mathrm{~mm}$ relative to bregma (adapted from Paxinos and Franklin (2001). The large box indicates the regions shown in $\boldsymbol{C}$ and $\boldsymbol{D}$, whereas the small box represents the region shown in $\boldsymbol{E}$. $\boldsymbol{C}, \boldsymbol{D}$, Double NA in the left side of a coronal section with low magnification, Cre recombinase expression, Mecp2 expression, and DAP WL of the same field with high magnification. E, BLA containing GFP epifluorescence was laser microdissected out (top) and after (bottom) laser microdissection. A microdissected region was traced with broken lines. $F, G$ rRNA. ${ }^{*} p<0.05$ by Student's $t$ test; AAV-GFP, $n=13$; AAV-CreGFP, $n=10$. Ctx, Cortex; Hip, hippocampus; CuP, caudate putaman; Thal, thalamus; Pir, piriform cortex.
$1 E)$. Similar to the FISH data, Cre expression was virtually absent in AAV-GFP-injected mice, whereas there was significant expression of Cre mRNA in AAV-CreGFP-injected mice (Fig. $1 F$ ). In these same animals, Mecp2 expression in the BLA was significantly reduced ( $~ 50 \%)$ in AAV-CreGFP-injected mice compared with AAV-GFPinjected mice, confirming the deletion of Mecp2 in the BLA (Fig. $1 G$ ). We found that within an infected cell, the deletion of Mecp2 was nearly 100\%; however, the reduction of Mecp2 expression within the entire BLA was 50\%, a "knockdown". Within this study, the AAVCreGFP-injected mice with reduced Mecp2 expression in the BLA will be referred to as localized knockdown or mice with knockdown of Mecp2 expression.

We also examined protein levels of Mecp2 in the BLA after the viral injections. We found that $\mathrm{MeCP} 2$ protein expression was sig- 
A
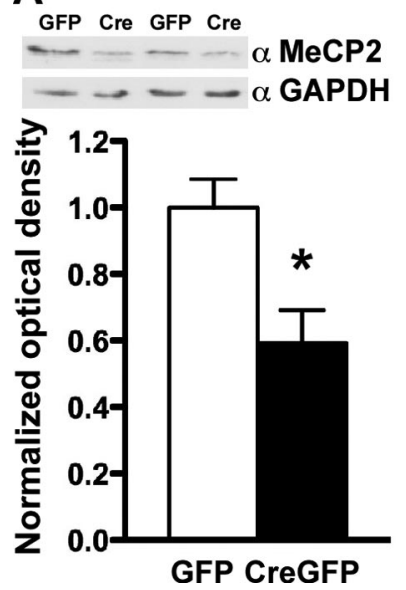

B
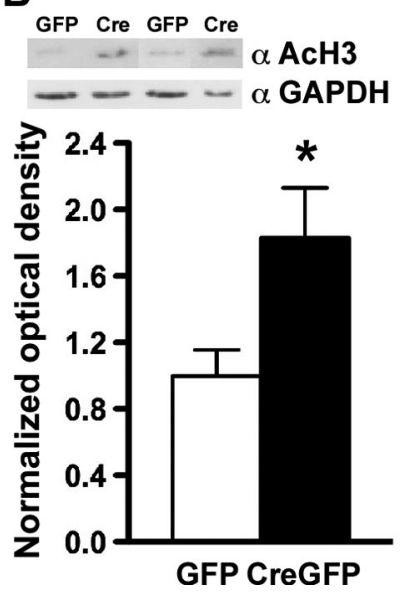

Figure 2. AAV-CreGFP-mediated deletion of Mecp2 from BLA neurons results in an increase in active gene transcription. Protein was extracted from the BLA region of AAV-CreGFP- and AAV-GFP-injected mice and subjected to Western blot analyses. A, AAV-CreGFP in the BLA produced a decrease ( $\sim 50 \%)$ in MeCP2 protein expression. $\boldsymbol{B}$, Western blot analysis revealed an increase in AcH3 levels in AAV-CreGFP-injected mice compared with AAV-GFP. Optical density of MeCP2 and AcH3 was normalized to that of GAPDH. * $p<0.05$ by Student's $t$ test; AAV-GFP, $n=5$; AAV-CreGFP, $n=5$ for $\boldsymbol{A}$ and $\boldsymbol{B}$.

nificantly reduced $(\sim 50 \%)$ in AAV-CreGFP-injected animals compared with the AAV-GFP controls (Fig. 2A). Because MeCP2 has been widely suggested to be a transcriptional repressor, we examined whether the localized knockdown of Mecp2 in the BLA would alter levels of acetylated histone $\mathrm{H} 3$ and histone $\mathrm{H} 4$, markers of active gene transcription. We found that the loss of Mecp2 produced a significant increase in levels of acetylated H3 (Fig. $2 \mathrm{~B}$ ), suggesting an upregulation of gene expression similar to that in a previous report (Shahbazian et al., 2002a). However, we did not detect a change in $\mathrm{H} 4$ acetylation levels (data not shown), suggesting that the loss of $\mathrm{MeCP} 2$ is mediating selective changes on chromatin.

\section{BLA-specific knockdown of Mecp2 results in increased anxiety-like behavior and deficits in cue-dependent fear conditioning}

To examine whether the localized knockdown of Mecp2 in the BLA would recapitulate any of the behavioral phenotypes observed in RTT patients, we tested these mice in several behavioral paradigms. By gross examination, AAV-CreGFP-injected mice were indistinguishable from AAV-GFP-injected animals, and there was no difference in weight between the two groups (data not shown). We used the rotarod test to examine whether selective deletion of Mecp2 in the BLA produces alterations in motor coordination since the conditional Mecp $2 \mathrm{KO}$ mice displayed significant deficits in this paradigm (Gemelli et al., 2006). Animals receiving AAV-CreGFP stayed on the rotarod for a similar amount of time over eight trials compared with AAV-GFPinjected mice, suggesting that Mecp2 knockdown in the BLA does not interfere with gross motor movement (Fig. 3A). Consistent with this finding, the BLA regionally specific Mecp2 knockdown mice did not display any signs of hindlimb clasping (data not shown), which has been reported with both the constitutive and conditional Mecp2 knock-outs (Chen et al., 2001; Guy et al., 2001; Gemelli et al., 2006). We next assessed locomotor activity during a 60 min testing period and found that total ambulation was indistinguishable between AAV-GFP- and AAV-CreGFPinjected animals, similar to the normal locomotor response reported with the conditional Mecp2 knock-outs (Fig. 3B).

Conditional Mecp2 KO mice, as well as a line with a truncating mutation in the Mecp2 gene $\left(M e c p 2^{308}\right)$, show elevated anxietyrelated behavior (Gemelli et al., 2006; McGill et al., 2006). Therefore, we examined whether the loss of Mecp2 in the BLA would produce alterations in anxiety-related behavior. We tested mice in the elevated-plus maze and the open-field test, behavioral paradigms that incorporate aspects of human anxiety and have predictive validity for anxiolytic drugs (Holmes, 2001; Crawley, 2008). In the elevated-plus maze, the knockdown of Mecp 2 in the BLA resulted in a significant increase $(p<0.05)$ in the time the animal spent in the closed arms, and a strong trend toward less time spent in the open arms, compared with control animals, suggestive of an increase in anxiety-related behavior (Fig. 3C). Consistent with this result, AAV-CreGFP mice showed a significant increase in latency to enter the open arm $(p<0.05)$ and a strong trend in latency to enter the closed arm compared with AAV-GFP-injected animals (Fig. 3D). In the open-field test, mice injected with AAV-CreGFP displayed a trend toward less time spent in the center field compared with AAV-GFP controls (Fig. $3 E$ ). More importantly, localized knockdown of Mecp2 in BLA resulted in a significant decrease in duration ratio of time spent in the complete center to that in periphery (Fig. $3 F$ ), as well as a significant increase in latency to enter the center field (Fig. 3G), suggestive of heightened anxiety-like behavior. Additionally, AAV-CreGFP-injected mice showed a strong trend toward less frequency to enter the center field (Fig. $3 H$ ). Collectively, these data show that the loss of Mecp2 in the BLA results in an increase in anxiety-related behavior.

We investigated whether the loss of Mecp2 in the BLA produces learning and memory deficits by examining both contextual and cued fear conditioning. Context-dependent fear conditioning requires the hippocampus and amygdala, whereas cuedependent fear requires the amygdala (LeDoux, 2000; Maren, 2001). In the fear-conditioning test, mice learn to associate a novel environment (chamber) or cue (auditory tone, $90 \mathrm{~dB}, 2.8$ $\mathrm{kHz}, 30 \mathrm{~s})$ with a footshock $(0.5 \mathrm{~mA}, 2 \mathrm{~s})$ after two pairings. We found the baseline freezing was indistinguishable between AAVGFP- and AAV-CreGFP-injected animals (data not shown). Testing the animals $24 \mathrm{~h}$ after training to assess contextdependent fear conditioning, we did not find a significant difference in the amount of freezing between the groups, although a trend toward a deficit was observed in the AAV-CreGFP mice similar to the trend observed with the conditional Mecp 2 knockouts (Gemelli et al., 2006) (Fig. 4A). Four hours after the contextual test, we examined cue-dependent fear conditioning and found that AAV-CreGFP-injected mice had a significant reduction in freezing $(p<0.05)$ compared with AAV-GFP-injected control mice, indicating a learning and memory deficit (Fig. 4A). This behavioral deficit was similar to that previously observed in the conditional Mecp2 KO mice (Gemelli et al., 2006). We also examined whether the AAV-GFP- and AAV-CreGFP-injected mice had similar responses to footshock at varying intensity (up to $0.25 \mathrm{~mA}$ ). We found no differences between the minimal current intensity to elicit flinch or jump responses in the AAV-GFP- or AAV-CreGFPinjected mice, suggesting that deficits in freezing were not caused by altered pain sensitivity between the groups (Fig. 4B).

We next tested the mice in a social interaction test since social deficits have been reported in mice with impaired Mecp2 function (Shahbazian et al., 2002a; Moretti et al., 2005; Gemelli et al., 2006). In this paradigm, an experimental animal is introduced to an open-field arena in which a wire mesh cage, either containing (social target) or lacking (no target) a C57BL/6 target mouse, is located at one end of the arena. We determined the amount of 
time the experimental animal spent in the interaction zone, defined as the area around the wire mesh cage (Fig. 4C). The data, expressed as a ratio of the time spent in the interaction zone with the social target to the time without the target, revealed no difference between AAV-GFP and AAV-CreGFP mice, suggesting that the knockdown of Mecp2 in the BLA does not alter social interaction behavior (Fig. 4D). Importantly, these data suggest that previously observed social interaction deficits by the loss of Mecp2 in the forebrain can be dissociated from the anxiety phenotype. In addition, animals receiving off-target placements of AAV-CreGFP (either one or both sides of the injection occurred outside of the BLA) did not display the increased anxiety or cue-dependent fearconditioning deficits observed in the AAV-CreGFP-injected BLA mice (supplemental Table 1, available at www.jneurosci. org as supplemental material), further strengthening our data that the loss of Mecp 2 in the BLA was mediating these behavioral phenotypes.

A recent study suggests that enhanced anxiety-like behavior in the Mecp $2^{308 / Y}$ RTT mouse model is associated with increased corticotropin-releasing hormone $(\mathrm{Crh})$ in the paraventricular nucleus of the hypothalamus, the central amygdala, and the bed nucleus of the stria terminalis (McGill et al., 2006). We therefore examined Crh mRNA expression in the BLA of mice with AAV-CreGFP injections to produce the region-specific Mecp2 knockdown to see whether altered expression of Crh may be involved in the alteration in anxiety-related behavior. However, we did not find any significant difference in $\mathrm{Crh}$ expression relative to AAV-GFP-injected mice, although a trend toward decreased expression was observed (supplemental Fig. 1, available at www.jneurosci.org as supplemental material). Interestingly, recent reports have shown that Crh expression is significantly downregulated in the hypothalamus of MeCP2 null KOs, as well as in the paraventricular nucleus of the hypothalamus of conditional $\mathrm{KO}$ mice in which MeCP2 was selectively deleted in Sim-1-expressing neurons in the hypothalamus (Chahrour et al., 2008; Fyffe et al., 2008). We also examined the expression of serum glucocorticoid-inducible kinase $1(S g k)$ and FK506-binding protein $51(F k b p)$, two glucocorticoid-regulated genes that have been shown to be upregulated in MeCP2 null mice (Nuber et al., 2005), gephyrin (Gphn) and $\alpha$-actinin $(A c t n)$, genes that have been implicated to play an important role in the amygdala after fear conditioning (Ressler et al., 2002; Mei et al., 2005), and brain-derived neurotrophic factor
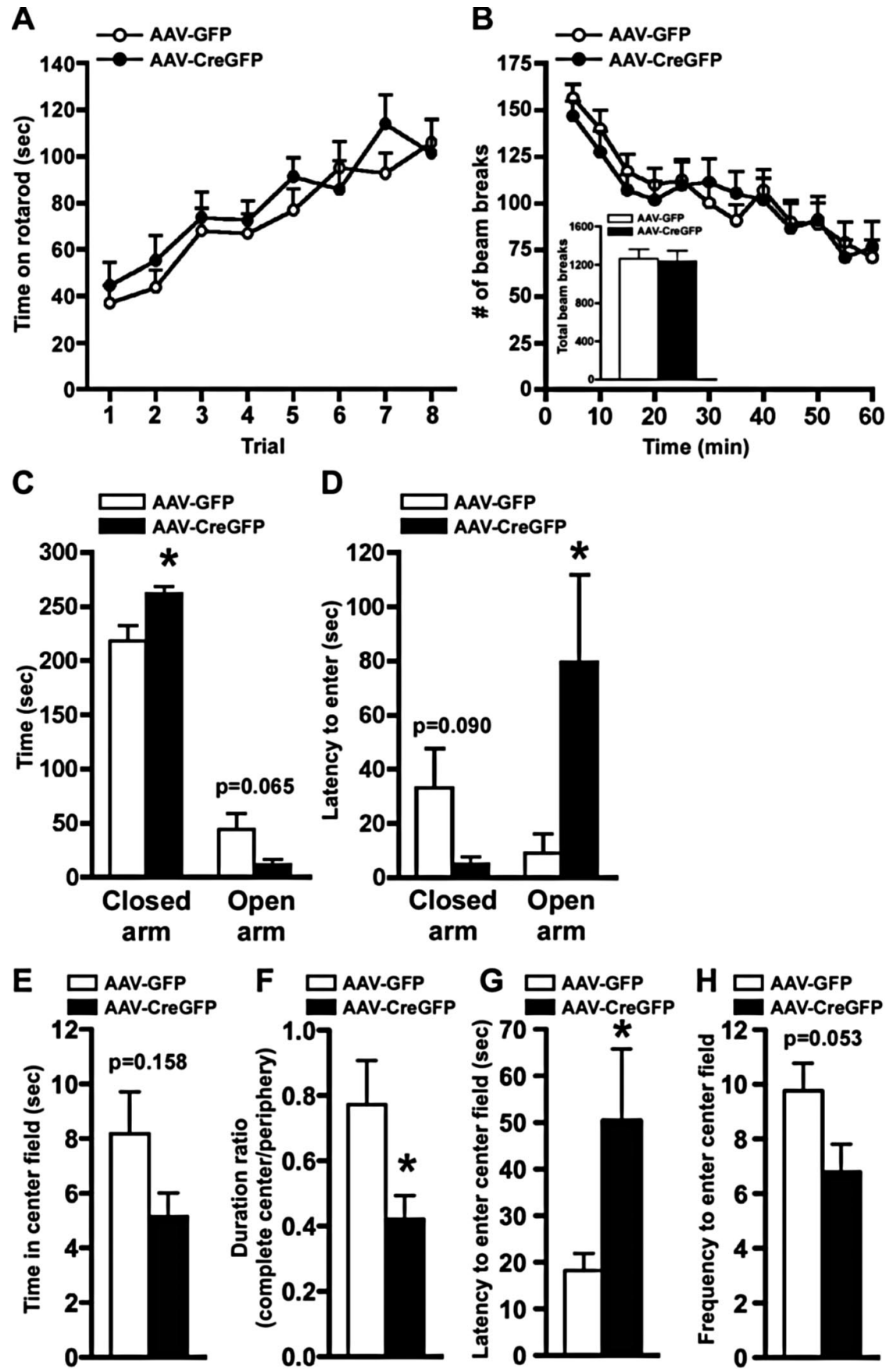

Figure 3. BLA-specific deletion of Mecp2 results in elevated anxiety-like behavior with normal locomotion and motor coordination. $\boldsymbol{A}$, AAV-CreGFP mice displayed motor coordination indistinguishable from that of AAV-GFP-injected mice on the rotarod test through eight trials. $B$, Locomotor activity was assessed by consecutive horizontal beam breaks for a 60 min testing period in 5 min increments. Inset, Total numbers of beam breaks during the test period were similar between AAV-GFP- and AAV-CreGFPinjected mice. $\boldsymbol{C}, \boldsymbol{D}$, Localized knockdown of Mecp 2 in the BLA resulted in heightened anxiety-like behavior. In the elevated-plus maze test, the localized deletion of Mecp2 in the BLA resulted in a significant increase in the time spent in the closed arm ( $p<$ $0.05)(\boldsymbol{C})$ as well as a significant increase in the latency to enter the open arm $(p<0.05)(\boldsymbol{D})$, indicating an increase in anxietyrelated behavior. $\boldsymbol{E}-\boldsymbol{H}, \mathrm{BLA}$-specific Mecp2 knockdown mice were assessed for the open-field test. Presented are time in center field $(\boldsymbol{E})$, duration ratio of time spent in complete center field to that to periphery $(\boldsymbol{F})$, latency to enter to center field $(\boldsymbol{G})$, and frequency to enter center field $(\boldsymbol{H})$. Localized knockdown of Mecp2 in BLA resulted in a significant decrease in duration ratio and a significant increase in latency to enter the center field compared with AAV-GFP mice ( $p<0.05)$, further supporting an increase in anxiety-related behavior. ${ }^{*} p<0.05$ by Student's $t$ test; AAV-GFP, $n=13$; AAV-CreGFP, $n=10$ for $\boldsymbol{A}-\boldsymbol{H}$.

$(B d n f)$, which has been suggested to be repressed and activated by MeCP2 (Chen et al., 2003; Martinowich et al., 2003; Chahrour et al., 2008). We did not detect a significant change in mRNA expression in any of the genes observed. Our data suggest mechanistically that 
A

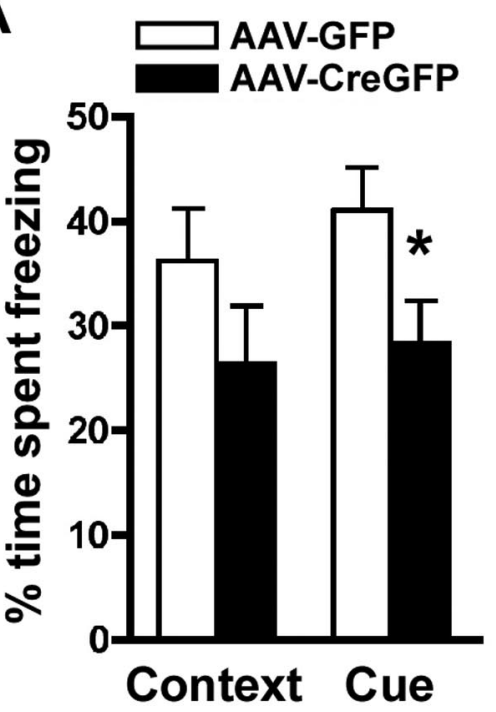

C

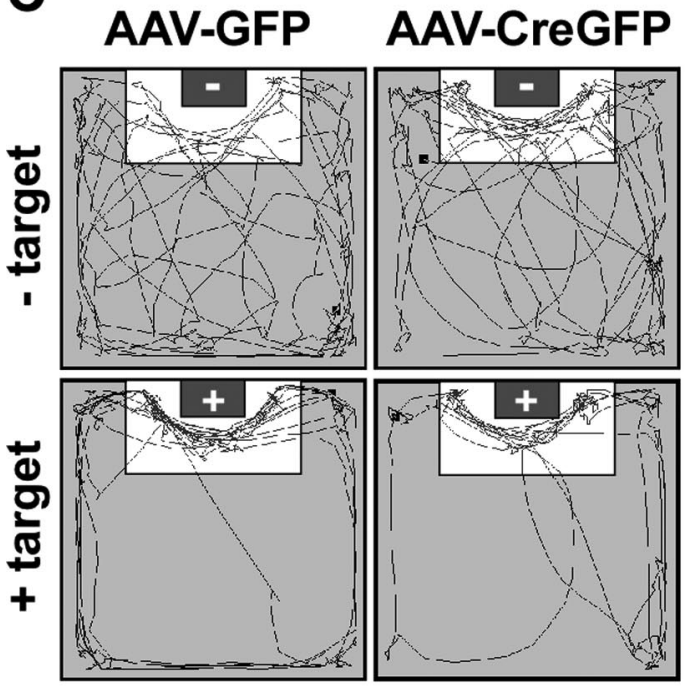

B

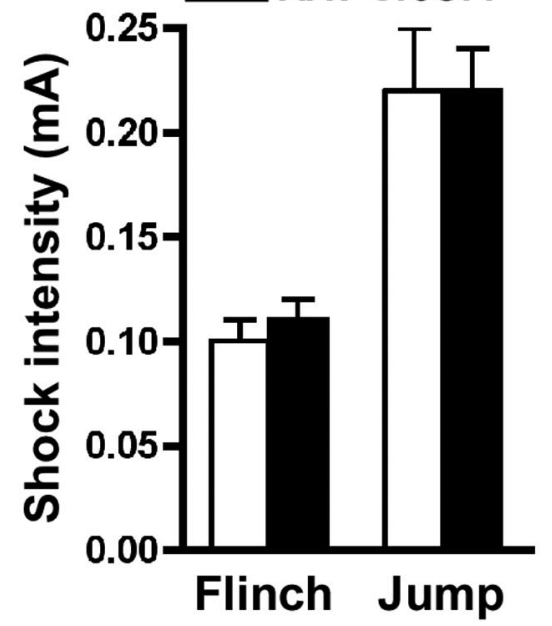

D

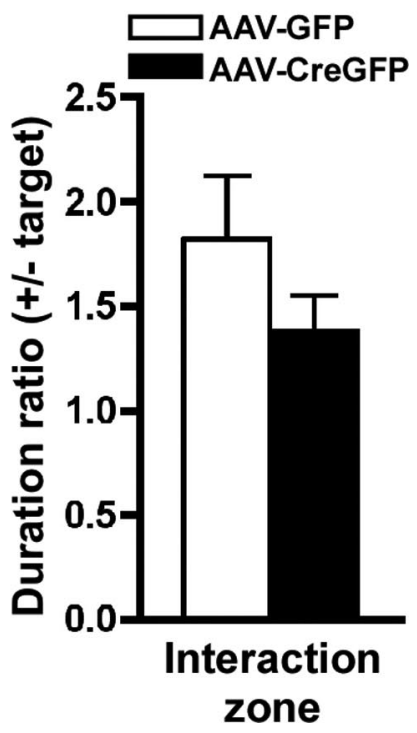

Figure 4. BLA-specific deletion of Mecp2 impairs cue-dependent fear conditioning. $\boldsymbol{A}$, The average amount of time spent freezing was determined for contextual and cue-dependent memory. AAV-CreGFP mice had a significant impairment $(p<0.05)$ in cue-dependent fear conditioning as demonstrated by a significant decrease in time spent freezing compared with AAV-GFP mice. There was no significant difference in context-dependent fear conditioning between AAV-GFP- and AAV-CreGFP-injected mice. B, AAV-GFP and AAV-CreGFP mice had similar footshock sensitivity to pain as measured by shock intensity to elicit flinch and jump responses. $\boldsymbol{C}$, Representative video tracking data from the social interaction test. The gray box in the arena indicates the location of the target mouse. - and + in the gray boxes indicate the absence or presence of the target mouse, respectively. $\boldsymbol{D}$, The ratio of time spent in the interaction zone with the target to the time without the target revealed similar levels of social interaction between the AAV-GFP and AAV-CreGFP mice. ${ }^{*} p<0.05$ by Student's $t$ test; AAV-GFP, $n=13$; AAV-CreGFP, $n=10$ for $\boldsymbol{A}-\boldsymbol{E}$.

dysregulation of gene expression underlies the behavioral phenotype produced by the loss of Mecp 2 in the BLA; however, genes other than those examined appear to be involved in mediating this phenotype.

\section{HDAC inhibition in the BLA produces a behavioral phenotype} similar to the localized knockdown of Mecp2 in the BLA

Because the loss of Mecp2 in the BLA increased levels of acetylated $\mathrm{H} 3$, as well as recapitulating some behavioral features of RTT, we were interested to determine whether the behavioral abnormalities were caused by impaired transcriptional repression. Previous studies using DNA microarray analysis have found only subtle changes in gene expression after the loss of Mecp2 (Tudor et al.,
2002). Therefore, to determine whether the behavioral deficits after the loss of Mecp2 in the BLA were the result of impaired transcriptional repression, we took a pharmacological approach. We infused SAHA, an inhibitor of both class I and II HDACs, bilaterally into the BLA of wildtype $\mathrm{C} 57 \mathrm{BL} / 6$ mice via osmotic minipumps for $14 \mathrm{~d}$. Infusion of an HDAC inhibitor should result in more active gene expression, which we hypothesized would be similar to the loss of $\mathrm{MeCP} 2$ function observed in the localized BLA knockdown mice. After $7 \mathrm{~d}$ of chronic infusion of either SAHA or vehicle, the behavioral testing of the animals commenced (Fig. 5A). At day 14, the animals were killed and the brains were collected to determine the placement of the cannulas (Fig. 5B). Animals with placements outside the BLA were excluded from the behavioral data analysis. To confirm the infusion of the drug, we prepared protein extracts from the BLA of animals and examined the levels of acetylated histone $\mathrm{H} 3$ and $\mathrm{H} 4$. We found that SAHA-infused animals had a significant induction of global acetylation of histone $\mathrm{H} 3$ and $\mathrm{H} 4$ compared with those infused with vehicle, indicating an elevation in active gene expression (Fig. 5C,D).

We examined the chronic SAHA- or vehicle-infused BLA mice in several behavioral paradigms. We found that locomotor activity was unaltered after SAHA infusion compared with control mice receiving vehicle infusion (Fig. 6A). To assess anxiety-like behavior, the mice were tested in the open-field test (Fig. 6F). We found that SAHA-infused animals spent significantly less time in the center $(p<$ $0.05)$ and entered the center area significantly less frequently $(p<0.05)$ than those infused with vehicle (Fig. 6B,E). Consistent with this, the parameter expressed as a duration ratio of time spent in the complete center to that in periphery revealed that chronic SAHA infusion resulted in a significant decrease in the duration ratio compared with the control mice infused with vehicle (Fig. 6C). Additionally, the mice infused with SAHA displayed a strong trend toward an increase in latency to enter the center field. Taken together, these results from the open-field test indicate increased anxiety-like behavior similar to that observed in the mice with the localized deletion of Mecp2 in the BLA (Fig. $3 E-H)$. In the fear-conditioning paradigm, mice infused with the HDAC inhibitor displayed significant impairments in cuedependent memory, but only a trend in contextual fearconditioning memory (Fig. $6 G$ ), similar to that observed with the AAV-CreGFP-induced BLA knockdowns (Fig. 4A). We examined social interactions and did not find any social deficits in SAHA-infused mice compared with vehicle-infused animals (Fig. $6 \mathrm{H})$, in agreement with our findings with the BLA localized 
A

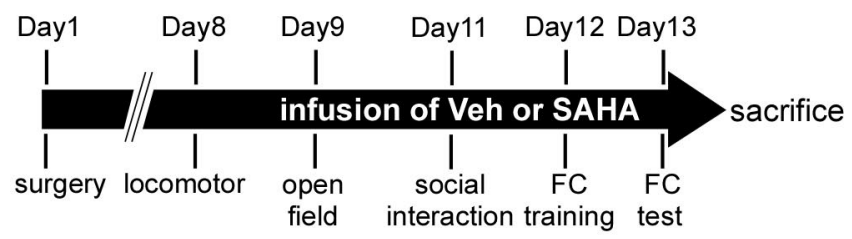

B

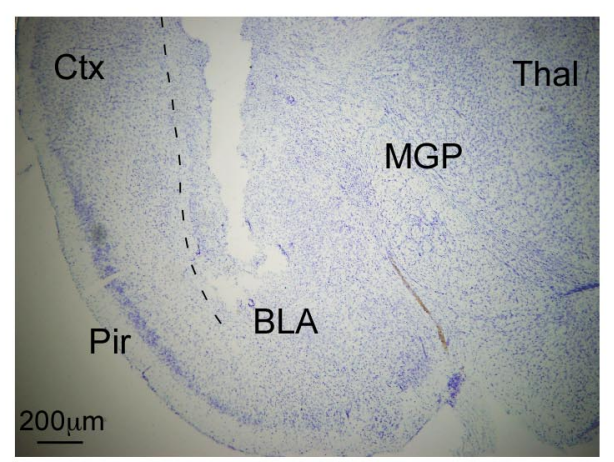

C
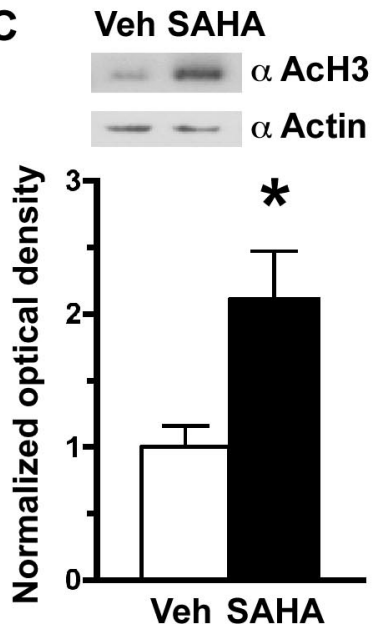

D Veh SAHA

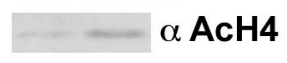
$-\alpha$ Actin

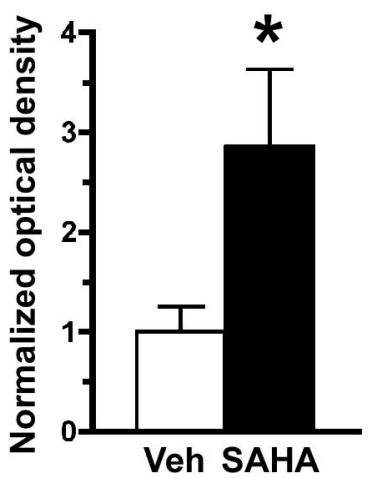

Figure 5. Chronic infusion of an $\mathrm{HDAC}$ inhibitor into the $\mathrm{BLA}$ of $\mathrm{C} 57 \mathrm{BL} / 6$ mice. $\boldsymbol{A}$, Timeline of the experiment. Mice were infused for $14 \mathrm{~d}$ with Vehicle (Veh) or SAHA bilaterally into the BLA using osmotic pumps. $\boldsymbol{B}$, An example of a Nissl-stained coronal brain section (left side) of the mouse through the track of a cannula. $\boldsymbol{C}, \boldsymbol{D}$, At the end of the experiment the animals were killed, placement of the cannula was confirmed, and then the BLA was dissected, and protein was isolated from the samples. Western blot analyses revealed a significant hyperacetylation of histone $3(\boldsymbol{C})$ and histone $4(\boldsymbol{D})$ in SAHA-infused animals. Top, Representative pictures of the Western blots. Bottom, Quantitation of the Western blots. Optical density of acetylated histone was normalized to that of actin * $p<0.05$ by Student's $t$ test; Veh, $n=10$; SAHA, $n=10$ for $C$ and $\boldsymbol{D}$. Ctx, Cortex; Thal, thalamus; MGP, medial globus pallidus; Pir, piriform cortex.

knockdowns (Fig. 4D), further indicating that heightened anxiety does not underlie the social interaction deficits observed in other mutant mouse models of RTT (Shahbazian et al., 2002a; Moretti et al., 2005; Gemelli et al., 2006). Furthermore, animals receiving off-target placements of SAHA infusions (either one or both sides of the injection occurred outside the BLA) did not display the increased anxiety or cuedependent fear-conditioning deficits observed in the SAHAinfused BLA mice (supplemental Table 2, available at www.jneurosci.org as supplemental material), further demonstrating that transcriptional repression in the BLA was involved in mediating these behavioral phenotypes.

\section{Discussion}

Collectively, our results show that intact Mecp2 function in the BLA is required for normal anxiety behavior as well as some types of learning and memory. Moreover, data from the HDAC inhibitor infusion experiment are consistent with the idea that Mecp2 acting as a transcriptional repressor in the BLA mediates these behavioral processes. Our ability to delete Mecp2 in adult brain and recapitulate behavioral phenotypes of RTT suggests a critical role for Mecp2 in mature neurons after neurodevelopment, in agreement with recent work demonstrating that the loss of $\mathrm{MeCP} 2$ in mature neurons produces significant deficits in synaptic transmission (Nelson et al., 2006). Furthermore, these data support the notion that Mecp 2 acts in a cell-autonomous manner in that upregulation of genes specifically in the BLA could account for specific phenotypes associated with the disease.

The current study builds on previous work demonstrating that the loss of Mecp2 function is sufficient to recapitulate aspects of RTT in mice (Chen et al., 2001; Guy et al., 2001; Shahbazian et al., 2002a; Gemelli et al., 2006; Pelka et al., 2006; Stearns et al., 2007). In this study, we extended these findings by demonstrating an essential role for Mecp2 in a particular brain region, the BLA, for mediating behavioral phenotypes associated with RTT. We found that the selective knockdown of Mecp2 in the BLA is sufficient to produce heightened anxiety-like behavior. We demonstrated that the infusion of an HDAC inhibitor in the BLA of wild-type mice resulted in a similar anxiety phenotype consistent with the idea that the deficit may be caused by alterations in gene expression. Previous studies examining conditional Mecp2 KO mice, as well as mice with a truncated mutation, $M e c p 2^{308 / Y}$, have shown that alterations in expression of Mecp2 can produce elevated anxiety-like behavior (Shahbazian et al., 2002; Gemelli et al., 2006; McGill et al., 2006). Studies examining Mecp2 male hypomorph mice, in which MeCP2 expression is at $50 \%$ of that of wild-type mice, have yielded conflicting data on anxiety-related behavior (Kerr et al., 2008; Samaco et al., 2008). Behavioral characterization of hemizygous males, which have a complete loss of MeCP2, have reported decreased levels of anxiety; however, these mice have growth and viability issues that require them to be tested at early developmental ages (Pelka et al., 2006; Stearns et al., 2007).

It appears that the stable disruption of the MeCP2 gene is important in influencing anxiety behavior as well as other neurological phenotypes. A recent study has shown that a transient disruption of MeCP2 in the amygdala at postnatal day $0-2$ was not sufficient to alter anxiety-related behavior in young adults (Kurian et al., 2008). In contrast, we deleted MeCP2 in adult animals such that the expression of MeCP2 remains knocked down and found increased anxiety-like behavior as well as cuedependent fear-conditioning deficits. The difference between these two studies is in line with recent findings demonstrating that reintroduction of $\mathrm{MeCP} 2$ in adult $\mathrm{MeCP} 2$ null mice can reverse the neurological deficits (Guy et al., 2007). Therefore, we believe the anxiety-like behavior, as well as other neurological phenotypes, is associated with concurrent loss of MeCP2.

One intriguing finding of the present study is that the suppression of Mecp2 in the BLA of mice, or the infusion of the HDAC inhibitor, was not sufficient to recapitulate the social interaction deficits observed in RTT mouse models (Shahbazian et al., 2002a; Moretti et al., 2005; Gemelli et al., 2006). Our data indicate an important dissociation between anxiety-related behavior and social interaction deficits in these RTT mouse models. This suggests that the social interaction deficits, which are one of the most debilitating 


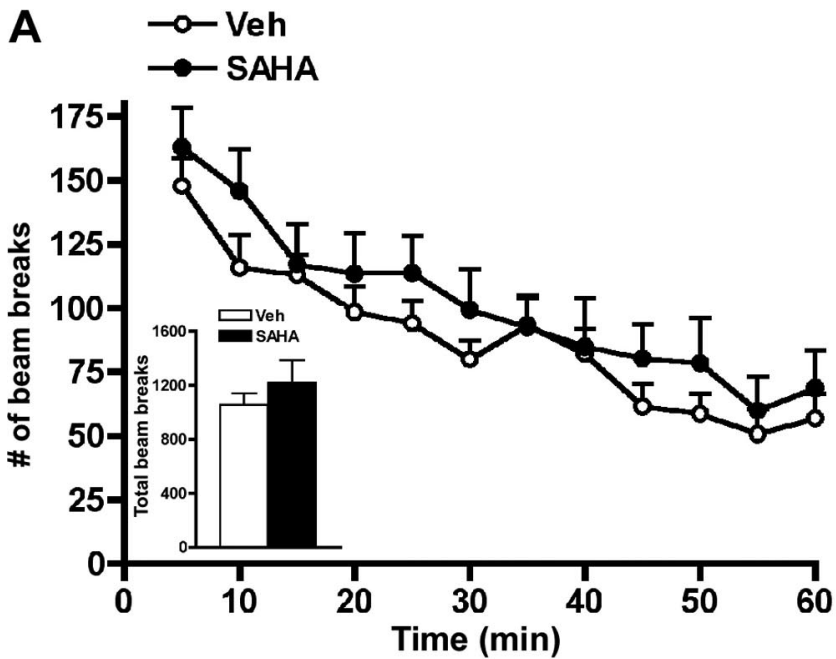

B

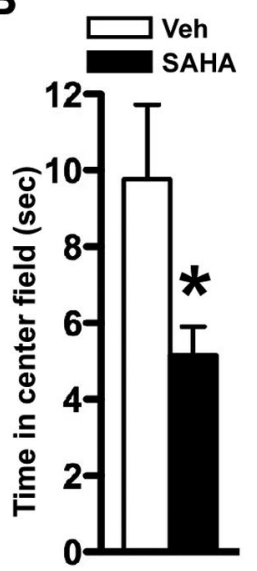

C

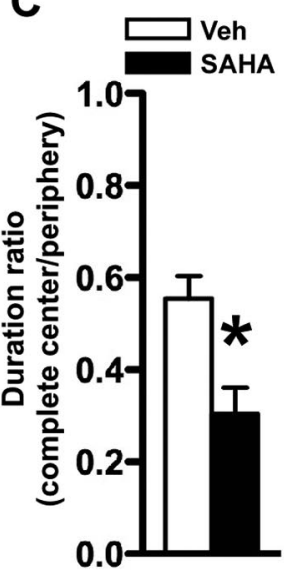

D

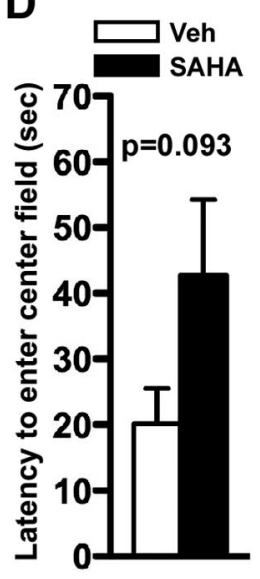

E
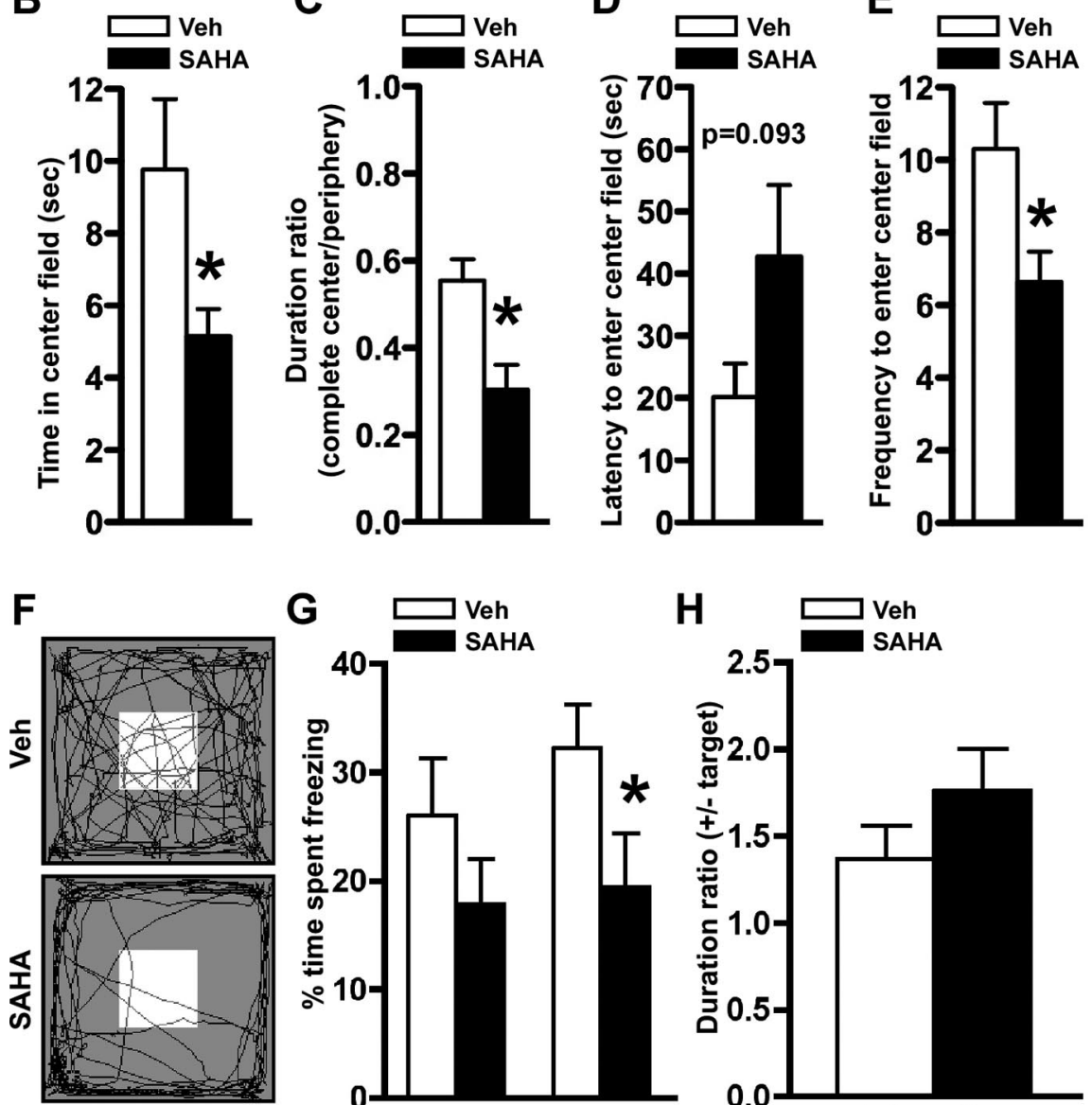

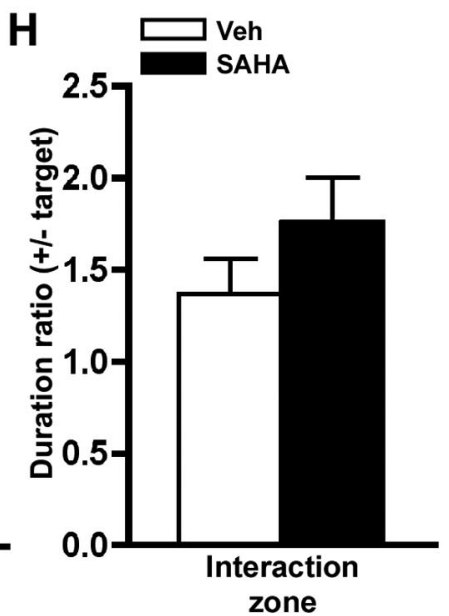

Figure 6. Mice chronically infused with SAHA in the BLA display behavioral phenotypes similar to the mice with BLA-specific deletion of Mecp2. A, Locomotor activity was unaltered by chronic SAHA infusions as assessed by consecutive horizontal beam breaks over a 60 min testing period when analyzed in 5 min increments or total beam breaks during the test period (inset). $\boldsymbol{B}-\boldsymbol{E}_{\text {, }}$ Chronic SAHA infusion resulted in increased anxiety-related behavior in the open-field test. Presented are time in center field $(\boldsymbol{E})$, duration ratio of time spent in complete center field to that to periphery $(\boldsymbol{F})$, latency to enter to center field $(\boldsymbol{G})$, and frequency to enter center field $(\boldsymbol{H})$. $\boldsymbol{F}$, Representative video tracking data from the open-field test. A white box in the arena indicates the center field. $\mathbf{G}$, In the fear-conditioning test, there was significantly less freezing in cue-dependent, but not contextual, memory formation from the mice infused with SAHA compared with those infused with vehicle (Veh). $\boldsymbol{H}$, Chronic SAHA infusions in the BLA did not alter social interaction as assessed by the ratio of time spent in the interaction zone with the target to the time without the target. ${ }^{*} p<0.05$ by Student's $t$ test; Veh, $n=10$; SAHA, $n=10$ for $\boldsymbol{A}-\boldsymbol{H}$. features of autism spectrum disorders, are not the result of heightened anxiety per se.

After local knockdown of Mecp2 in the BLA, a brain region required for cuedependent fear conditioning, we found cue-dependent fear-conditioning deficits similar to that observed with other Mecp2 mouse models of RTT (Gemelli et al., 2006; Pelka et al., 2006; Stearns et al., 2007; Samaco et al., 2008). This behavioral deficit was recapitulated in wild-type mice by chronically infusing an HDAC inhibitor in the BLA, suggesting that impaired transcriptional repression can impact specific forms of learning and memory. Interestingly, we did not see significant differences in contextual fear conditioning after the selective loss of Mecp2 in the BLA or infusion of the HDAC inhibitor in this brain region, although trends were observed similar to those reported for the conditional Mecp2 knock-out mice (Gemelli et al., 2006). The male hypomorph mice, which express MeCP2 at $50 \%$ of wild type, have been shown to have significant differences in context-dependent fear conditioning (Samaco et al., 2008). In male hemizygous mice, which have a complete loss of MeCP2, nonsignificant decreases as well as significant decreases on contextdependent fear conditioning have been reported (Pelka et al., 2006; Stearns et al., 2007). Studies examining contextual fear conditioning in mice with a truncated mutation, Mecp $2^{308 / Y}$, have reported normal as well as significantly decreased learning and memory (Shahbazian et al., 2002a; Moretti et al., 2006).

Recent work has suggested that acute systemic injection of an HDAC inhibitor, or acute localized infusion of an HDAC inhibitor to the CA1 subfield of the hippocampus, before contextual fear conditioning increases $\mathrm{H} 3$ acetylation transiently and enhances learning and memory (Levenson et al., 2004; Vecsey et al., 2007). The apparent disparity between these studies and the data presented here may be attributable to the chronic nature of our drug treatment compared with the acute administration in the previous work. It is possible that chronically elevated histone acetylation may lead to saturation of changes in neuronal plasticity compared with that observed by a single drug dose. This suggests that the therapeutic use of HDAC inhibitors may require further caution as they may produce nondesirable psychiatric behavioral side effects after chronic administration.

A recent study has suggested that Mecp2 may act as either a transcriptional activator or repressor in the hypothalamus (Chahrour et al., 2008). Although Mecp2 
may have dual functions as a transcriptional activator or repressor in hypothalamus, and perhaps in other brain regions, the similar behavioral phenotypes produced by the loss of Mecp2 in the BLA, and the infusion of the HDAC inhibitor, strongly suggests that these particular behavioral deficits may be caused by alterations in transcriptional repression in this brain region.

The viral-mediated knockdown approach used in this study to selectively impair Mecp2 function in the BLA establishes the importance of Mecp2 in this brain region in normal anxiety-related behavior and some forms of learning and memory. The similar behavioral phenotype observed with the infusion of the HDAC inhibitor in the BLA of wild-type mice suggests that these behavioral deficits are caused by impaired transcriptional repression resulting from loss of $\mathrm{MeCP} 2$ function, providing direct mechanistic insight into the RTT disease process. The ability to recapitulate bona fide deficits of the RTT phenotype in mice is important for understanding and ultimately developing treatment advances for these components of the disorder.

\section{References}

Adachi M, Keefer EW, Jones FS (2005) A segment of the Mecp2 promoter is sufficient to drive expression in neurons. Hum Mol Genet 14:3709-3722.

Adachi M, Barrot M, Autry AE, Theobald D, Monteggia LM (2008) Selective loss of brain-derived neurotrophic factor in the dentate gyrus attenuates antidepressant efficacy. Biol Psychiatry 63:642-649.

Amir RE, Van den Veyver IB, Wan M, Tran CQ, Francke U, Zoghbi HY (1999) Rett syndrome is caused by mutations in X-linked MECP2, encoding methyl-CpG-binding protein 2. Nat Genet 23:185-188.

Asaka Y, Jugloff DG, Zhang L, Eubanks JH, Fitzsimonds RM (2006) Hippocampal synaptic plasticity is impaired in the Mecp2-null mouse model of Rett syndrome. Neurobiol Dis 21:217-227.

Berton O, McClung CA, Dileone RJ, Krishnan V, Renthal W, Russo SJ, Graham D, Tsankova NM, Bolanos CA, Rios M, Monteggia LM, Self DW, Nestler EJ (2006) Essential role of BDNF in the mesolimbic dopamine pathway in social defeat stress. Science 311:864-868.

Campeau S, Davis M (1995) Involvement of the central nucleus and basolateral complex of the amygdala in fear conditioning measured with fearpotentiated startle in rats trained concurrently with auditory and visual conditioned stimuli. J Neurosci 15:2301-2311.

Chahrour M, Jung SY, Shaw C, Zhou X, Wong ST, Qin J, Zoghbi HY (2008) $\mathrm{MeCP} 2$, a key contributor to neurological disease, activates and represses transcription. Science 320:1224-1229.

Chen RZ, Akbarian S, Tudor M, Jaenisch R (2001) Deficiency of methylCpG binding protein-2 in CNS neurons results in a Rett-like phenotype in mice. Nat Genet 27:327-331.

Chen WG, Chang Q, Lin Y, Meissner A, West AE, Griffith EC, Jaenisch R, Greenberg ME (2003) Derepression of BDNF transcription involves calcium-dependent phosphorylation of MeCP2. Science 302:885-889.

Crawley JN (2008) Behavioral phenotyping strategies for mutant mice. Neuron 57:809-818.

Dani VS, Chang Q, Maffei A, Turrigiano GG, Jaenisch R, Nelson SB (2005) Reduced cortical activity due to a shift in the balance between excitation and inhibition in a mouse model of Rett syndrome. Proc Natl Acad Sci U S A 102:12560-12565.

Davis M, Shi C (2000) The amygdala. Curr Biol 10:R131.

Fyffe SL, Neul JL, Samaco RC, Chao HT, Ben-Shachar S, Moretti P, McGill BE, Goulding EH, Sullivan E, Tecott LH, Zoghbi HY (2008) Deletion of Mecp2 in Sim1-expressing neurons reveals a critical role for MeCP2 in feeding behavior, aggression, and the response to stress. Neuron 59:947-958

Gemelli T, Berton O, Nelson ED, Perrotti LI, Jaenisch R, Monteggia LM (2006) Postnatal loss of methyl-CpG binding protein 2 in the forebrain is sufficient to mediate behavioral aspects of Rett syndrome in mice. Biol Psychiatry 59:468-476.

Guy J, Hendrich B, Holmes M, Martin JE, Bird A (2001) A mouse Mecp2null mutation causes neurological symptoms that mimic Rett syndrome. Nat Genet 27:322-326.

Guy J, Gan J, Selfridge J, Cobb S, Bird A (2007) Reversal of neurological defects in a mouse model of Rett syndrome. Science 315:1143-1147.

Hagberg B, Aicardi J, Dias K, Ramos O (1983) A progressive syndrome of autism, dementia, ataxia, and loss of purposeful hand use in girls: Rett's syndrome: report of 35 cases. Ann Neurol 14:471-479.

Holmes A (2001) Targeted gene mutation approaches to the study of anxiety-like behavior in mice. Neurosci Biobehav Rev 25:261-273.

Kerr B, Alvarez-Saavedra M, Sáez MA, Saona A, Young JI (2008) Defective body weight regulation, motor control and abnormal social interactions in Mecp2 hypomorphic mice. Hum Mol Genet 17:1707-1717.

Kishi N, Macklis JD (2004) MECP2 is progressively expressed in postmigratory neurons and is involved in neuronal maturation rather than cell fate decisions. Mol Cell Neurosci 27:306-321.

Kurian JR, Bychowski ME, Forbes-Lorman RM, Auger CJ, Auger AP (2008) Mecp2 organizes juvenile social behavior in a sex-specific manner. J Neurosci 28:7137-7142.

LeDoux J (2007) The amygdala. Curr Biol 17:R868-R874.

LeDoux JE (2000) Emotion circuits in the brain. Annu Rev Neurosci 23:155-184.

Levenson JM, O’Riordan KJ, Brown KD, Trinh MA, Molfese DL, Sweatt JD (2004) Regulation of histone acetylation during memory formation in the hippocampus. J Biol Chem 279:40545-40559.

Maren S (2001) Neurobiology of Pavlovian fear conditioning. Annu Rev Neurosci 24:897-931.

Maren S, Fanselow MS (1995) Synaptic plasticity in the basolateral amygdala induced by hippocampal formation stimulation in vivo. J Neurosci 15:7548-7564.

Martinowich K, Hattori D, Wu H, Fouse S, He F, Hu Y, Fan G, Sun YE (2003) DNA methylation-related chromatin remodeling in activity-dependent BDNF gene regulation. Science 302:890-893.

McGill BE, Bundle SF, Yaylaoglu MB, Carson JP, Thaller C, Zoghbi HY (2006) Enhanced anxiety and stress-induced corticosterone release are associated with increased Crh expression in a mouse model of Rett syndrome. Proc Natl Acad Sci U S A 103:18267-18272.

Mei B, Li C, Dong S, Jiang CH, Wang H, Hu Y (2005) Distinct gene expression profiles in hippocampus and amygdala after fear conditioning. Brain Res Bull 67:1-12.

Monteggia LM, Barrot M, Powell CM, Berton O, Galanis V, Gemelli T, Meuth S, Nagy A, Greene RW, Nestler EJ (2004) Essential role of brain-derived neurotrophic factor in adult hippocampal function. Proc Natl Acad Sci U S A 101:10827-10832.

Moretti P, Bouwknecht JA, Teague R, Paylor R, Zoghbi HY (2005) Abnormalities of social interactions and home-cage behavior in a mouse model of Rett syndrome. Hum Mol Genet 14:205-220.

Moretti P, Levenson JM, Battaglia F, Atkinson R, Teague R, Antalffy B, Armstrong D, Arancio O, Sweatt JD, Zoghbi HY (2006) Learning and memory and synaptic plasticity are impaired in a mouse model of Rett syndrome. J Neurosci 26:319-327.

Mount RH, Charman T, Hastings RP, Reilly S, Cass H (2002) The Rett Syndrome Behaviour Questionnaire (RSBQ): refining the behavioural phenotype of Rett syndrome. J Child Psychol Psychiatry 43:1099-1110.

Mullaney BC, Johnston MV, Blue ME (2004) Developmental expression of methyl-CpG binding protein 2 is dynamically regulated in the rodent brain. Neuroscience 123:939-949.

Nelson ED, Kavalali ET, Monteggia LM (2006) MeCP2-dependent transcriptional repression regulates excitatory neurotransmission. Curr Biol 16:710-716.

Nelson ED, Kavalali ET, Monteggia LM (2008) Activity-dependent suppression of miniature neurotransmission through the regulation of DNA methylation. J Neurosci 28:395-406.

Nuber UA, Kriaucionis S, Roloff TC, Guy J, Selfridge J, Steinhoff C, Schulz R, Lipkowitz B, Ropers HH, Holmes MC, Bird A (2005) Up-regulation of glucocorticoid-regulated genes in a mouse model of Rett syndrome. Hum Mol Genet 14:2247-2256.

Paxinos G, Franklin KB (2001) The mouse brain in stereotaxic coordinates, Ed 2. San Diego: Elsevier Academic.

Pelka GJ, Watson CM, Radziewic T, Hayward M, Lahooti H, Christodoulou J, Tam PP (2006) Mecp2 deficiency is associated with learning and cognitive deficits and altered gene activity in the hippocampal region of mice. Brain 129:887-898.

Rainnie DG, Bergeron R, Sajdyk TJ, Patil M, Gehlert DR, Shekhar A (2004) Corticotrophin releasing factor-induced synaptic plasticity in the amygdala translates stress into emotional disorders. J Neurosci 24:3471-3479.

Ressler KJ, Paschall G, Zhou XL, Davis M (2002) Regulation of synaptic plasticity genes during consolidation of fear conditioning. J Neurosc 22:7892-7902. 
Roozendaal B, Brunson KL, Holloway BL, McGaugh JL, Baram TZ (2002) Involvement of stress-released corticotropin-releasing hormone in the basolateral amygdala in regulating memory consolidation. Proc Natl Acad Sci U S A 99:13908-13913.

Samaco RC, Fryer JD, Ren J, Fyffe S, Chao HT, Sun Y, Greer JJ, Zoghbi HY, Neul JL (2008) A partial loss of function allele of Methyl-CpG-Binding Protein predicts a human neurodevelopmental syndrome. Hum Mol Genet 17:1718-1727.

Sansom D, Krishnan VH, Corbett J, Kerr A (1993) Emotional and behavioural aspects of Rett syndrome. Dev Med Child Neurol 35:340-345.

Shahbazian M, Young J, Yuva-Paylor L, Spencer C, Antalffy B, Noebels J, Armstrong D, Paylor R, Zoghbi H (2002a) Mice with truncated MeCP2 recapitulate many Rett syndrome features and display hyperacetylation of histone H3. Neuron 35:243-254.

Shahbazian MD, Antalffy B, Armstrong DL, Zoghbi HY (2002b) Insight into Rett syndrome: MeCP2 levels display tissue- and cell-specific differences and correlate with neuronal maturation. Hum Mol Genet 11:115-124.
Stearns NA, Schaevitz LR, Bowling H, Nag N, Berger UV, Berger-Sweeney J (2007) Behavioral and anatomical abnormalities in Mecp 2 mutant mice: a model for Rett syndrome. Neuroscience 146:907-921.

Tudor M, Akbarian S, Chen RZ, Jaenisch R (2002) Transcriptional profiling of a mouse model for Rett syndrome reveals subtle transcriptional changes in the brain. Proc Natl Acad Sci U S A 99:15536-15541.

Vecsey CG, Hawk JD, Lattal KM, Stein JM, Fabian SA, Attner MA, Cabrera SM, McDonough CB, Brindle PK, Abel T, Wood MA (2007) Histone deacetylase inhibitors enhance memory and synaptic plasticity via CREB:CBP-dependent transcriptional activation. J Neurosci 27: $6128-6140$.

Weaving LS, Ellaway CJ, Gecz J, Christodoulou J (2005) Rett syndrome: clinical review and genetic update. J Med Genet 42:1-7.

Wilensky AE, Schafe GE, LeDoux JE (1999) Functional inactivation of the amygdala before but not after auditory fear conditioning prevents memory formation. J Neurosci 19:RC48:(1-5). 